Research article

urn:lsid:zoobank.org:pub:12228A56-6AA7-405F-8A8D-093988D00C1F

\title{
Three new species of Entomobrya (Collembola: Entomobryidae) from China
}

\author{
Yitong $\mathrm{MA}^{1, *}$ \& Shidi $\mathrm{SHI}^{2}$ \\ ${ }^{1}$ School of Life Science, Nantong University, Nantong, Jiangsu 226000, P. R. China. \\ ${ }^{2}$ School of Life Sciences, Taizhou University, Linhai, Zhejiang 317000, P. R. China. \\ ${ }^{*}$ Corresponding author: mayitong@ntu.edu.cn \\ 2Email: shiii@tzc.edu.cn \\ ${ }^{1}$ urn:1sid:zoobank.org:author:50F82475-5B63-461D-BC1D-555FE4BF7C09 \\ ${ }^{2}$ urn:1sid:zoobank.org:author:01C93AAA-7FF4-47E9-A024-17DEC7A116EE
}

\begin{abstract}
Three new species of Entomobrya Rondani, 1861 from China are described: E. leviseta sp. nov. and E. polychaeta sp. nov. from Shaanxi Province and E. dingi sp. nov. from Yunnan Province. This is the first report of Entomobrya from Shaanxi Province. Entomobrya leviseta sp. nov. is characterised by prelabral smooth chaetae on the labrum; E. polychaeta sp. nov. by three pairs of longitudinal dark blue stripes from Th. II to Abd. III and eight lateral mac on Abd. III; and E. dingi sp. nov. by only a little pigment on the body and 5 central mac on And. II \& III. A key to all Chinese species of Entomobrya is given.
\end{abstract}

Keywords. Entomobryinae, taxonomy, chaetotaxy.

Ma Y. \& Shi S. 2018. Three new species of Entomobrya (Collembola: Entomobryidae) from China. European Journal of Taxonomy 419: 1-21. https://doi.org/10.5852/ejt.2018.419

\section{Introduction}

Entomobryidae Tömösvary, 1882 is the largest family of Collembola Lubbock, 1873 and it contains seven subfamilies (Zhang \& Louis 2014) with about 1800 species recorded worldwide (Bellinger et al. 1996-2017). The genus Entomobrya, Rondani, 1861 contains 270 species and is one of the largest genera; it is characterised by four-segmented antennae, eyes $8+8$, scales absent, mucro bidentate with a basal spine, and dens without spine. Colour pattern sometimes play a key role in classification, although it may be variable among specimens from a single population (Stach 1963); The molecular phylogenetic study by Katz et al. (2015) revealed that colour pattern is a valid tool for specific delimitation in Entomobrya. Chaetotaxy has proved to be extremely important (Jordana 2012). The labrum, especially its papillae and prelabral setae, is also very important and other characteristics, such as the shape of the mucro, labium, and apical bulb of the antenna, are of occasional taxonomic value (Christiansen 1958).

Fifteen species of Entomobrya have been reported from China. Two new species, E. leviseta sp. nov. and E. polychaeta sp. nov., are found in Shaanxi and ours is the first report of the genus in the province. 
Another new species, E. dingi sp. nov., is found in Yunnan and this is the third species of the genus in the province. A key to the Chinese species of Entomobrya is given.

\section{Material and methods}

Specimens were cleared and mounted under a coverslip in Marc André II solution and were studied with a Leica DM2500 microscope. Photographs were taken with a mounted Leica DFC300 FX digital camera and enhanced with Photoshop CS2 (Adobe Inc.). The nomenclature of the dorsal chaetotaxy of the head is described following Jordana \& Baquero (2005) and Chen \& Christiansen (1993), and interocular chaetae after Mari-Mutt (1986). Labial chaetae are designated following Gisin (1967). Tergal chaetae of the body are designated using the system of Szeptycki (1979).

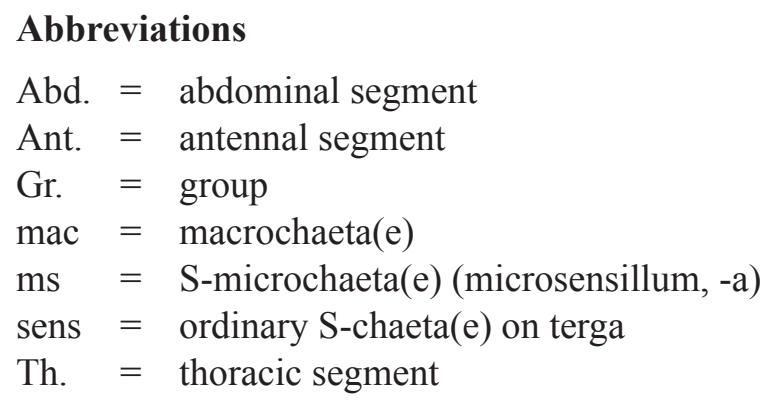

\section{Results}

Description of new species

Class Collembola Lubbock, 1873

Order Entomobryomorpha Börner, 1913

Family Entomobryidae Tömösvary, 1882

Subfamily Entomobryinae Schäffer, 1896

Genus Entomobrya Rondani, 1861

\section{Diagnosis}

Moderate size, usually 1-3 mm; antennae four segmented and usually with an apical bulb; eyes $8+8$; intersegmental membranes without microchaetae; scales absent and body chaetae not scale-like; mucro bidentate and with a basal spine; dens without spine.

Entomobrya leviseta sp. nov

urn:1sid:zoobank.org:act:5FE7323A-E49C-40D9-9576-6B82E3D4D355

Figs 1-5, Table 1

\section{Diagnosis}

Eyepatches and lateral margin of Th. II with blue pigment; a longitudinal blue stripe present along midline from head to Th. II, Th. III with a broad transverse blue stripe; Abd. IV with two irregular transverse blue stripes; prelabral chaetae of labrum smooth; subapical tooth of mucro equal to or slightly larger than apical tooth.

\section{Etymology}

The specific epithet is named after the smooth (from Latin 'levis') prelabral chaetae of the labrum. 
Table 1. Comparison between Entomobrya leviseta sp. nov and E. striatella Börner, 1909.

\begin{tabular}{lcc}
\hline \multicolumn{1}{c}{ Characters } & E. leviseta sp. nov. & E. striatella \\
\hline Labial chaetae & MREL $_{1} \mathrm{~L}_{2}$ & $\mathrm{M}_{1} \mathrm{M}_{2} \mathrm{REL}_{1} \mathrm{~L}_{2}$ \\
Transverse stripe on Th. III & broad & narrow \\
Colour pattern on Abd. IV anteriorly & a transverse stripe & a pair of patches \\
Colour pattern on Abd. V & a transverse stripe & a pair of patches \\
Mac m2i2 on Th. II & present & absent \\
Mac a1a on Abd. I & present & absent \\
Central mac on Abd. IV & $33-46$ & 24 \\
Locality & China (Shaanxi) & Japan, Korea \\
\hline
\end{tabular}

\section{Type material}

\section{Holotype}

CHINA: + on slide, 108 National Highway, Mazhao Town, Zhouzhi County, Xi'an City, Shaanxi Province, $34^{\circ} 03^{\prime} 35^{\prime \prime} \mathrm{N}, 108^{\circ} 11^{\prime} 47^{\prime \prime} \mathrm{E}$, altitude ca $566 \mathrm{~m}$, in litter, with aspirator, 15 Jul. 2012, Yitong Ma leg. (Nantong University, collection number 1115).

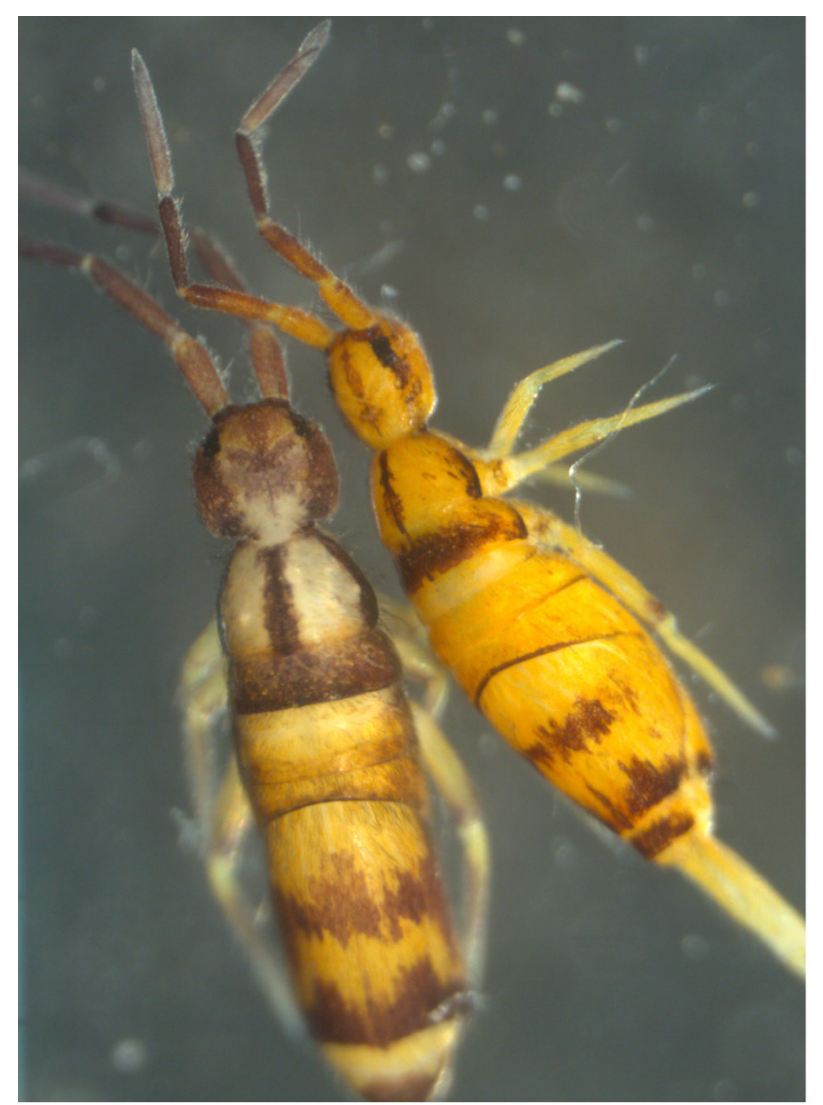

A

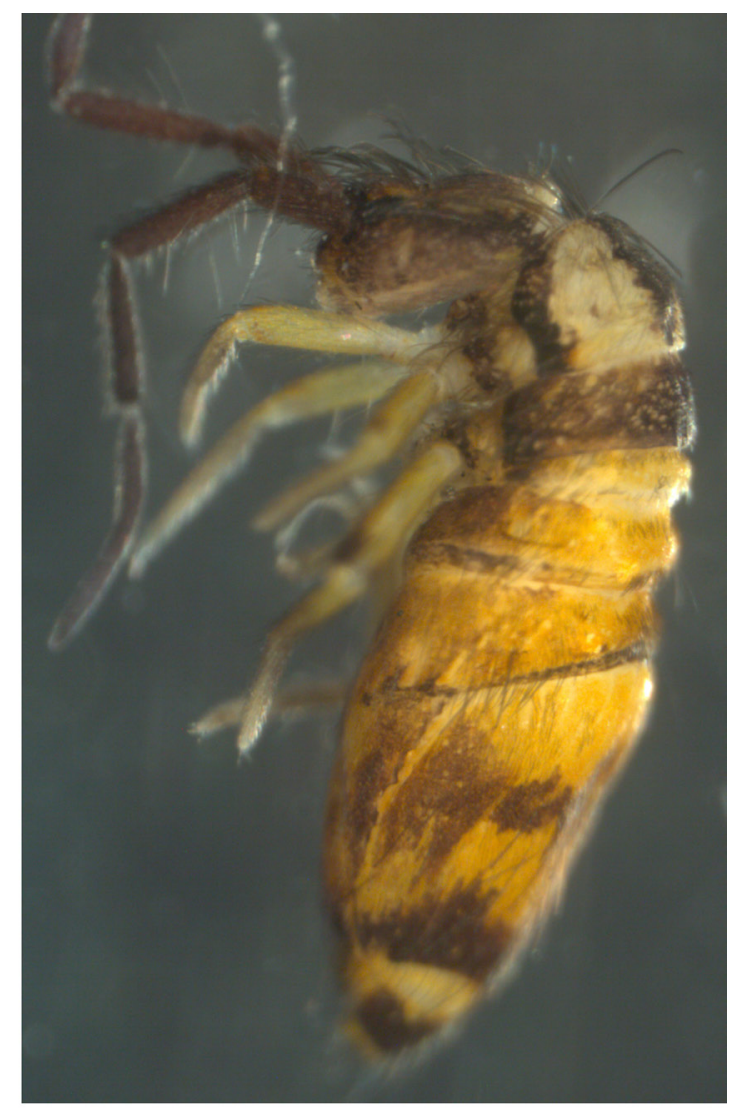

$\mathrm{B}$

Fig. 1. Entomobrya leviseta sp. nov. A-B. Habitus. Scales bars: A-B $=500 \mu \mathrm{m}$. 


\section{Paratypes}

CHINA: 2 우 on slides, same collecting data as for holotype; 5 우 on slides, Famen Temple, Fufeng

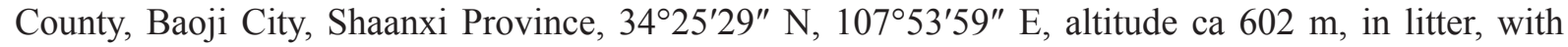
aspirator, 11 Jul. 2012, Yitong Ma leg. (Nantong University, collection number 1101).

\section{Description}

Measurements. Body length up to $2.68 \mathrm{~mm}$.

CoLour. Ground colour yellowish. Eyepatches dark blue. Antennae and lateral margin of head and Th. II with blue pigment. A longitudinal blue stripe present along midline from head to Th. II. Th. III with a broad transverse blue stripe. Abd. II with scattered pigment laterally and Abd. III with a very narrow blue stripe posteriorly. Abd. IV with two irregular transverse blue stripes. Coxae and tibiotarsi with weak pigment (Fig. 1A-B).

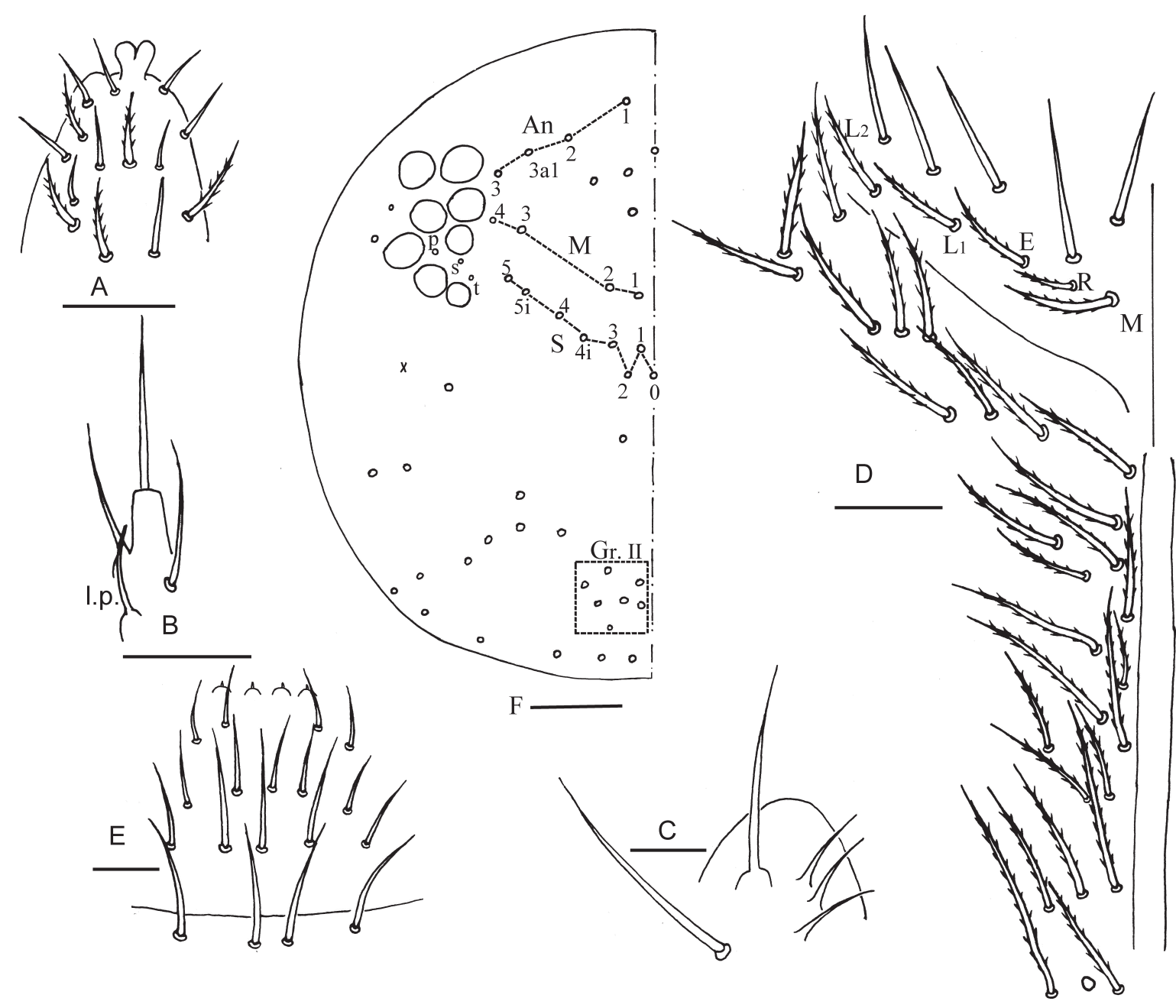

Fig. 2. Entomobrya leviseta sp. nov. A. Apex of Ant. IV. B. Lateral process of labial palp. C. Maxillary outer lobe. D. Labium. E. Labrum. F. Dorsal chaetotaxy of head. Scale bars: D, F = 50 $\mu$; A-C, E = $20 \mu \mathrm{m}$. 
HEAD. Antenna 0.47-0.63 times as long as body. Antennal segments ratio as I: II : III: IV $=1.00: 1.28-$ $1.72: 1.28-1.65: 1.84-2.57$. Distal part of Ant. IV with many sensory chaetae, normal ciliate chaetae and apical bulb bilobed (Fig. 2A). Lateral process (1.p.) of labial palp curved with tip not reaching apex of same papilla (Fig. 2B). Subapical chaeta of maxillary outer lobe subequal to apical one, 3 smooth hairs on sublobal plate (Fig. 2C). Labial chaetae $\mathrm{MREL}_{1} \mathrm{~L}_{2}$, all ciliate; chaeta $\mathrm{R}$ 0.56-0.63 times as long as chaeta M (Fig. 2D). Labral chaetae 4/5, 5, 4, all smooth; labral papillae 4 and each with 1 minute denticle (Fig. 2E). Clypeus not clearly seen. Dorsal cephalic chaetotaxy with 4 antennal (An), 4 median (M) and 8 sutural (S) mac, 7 mac in Gr. II, interocular chaetae as p, s, t (Fig. 2F).

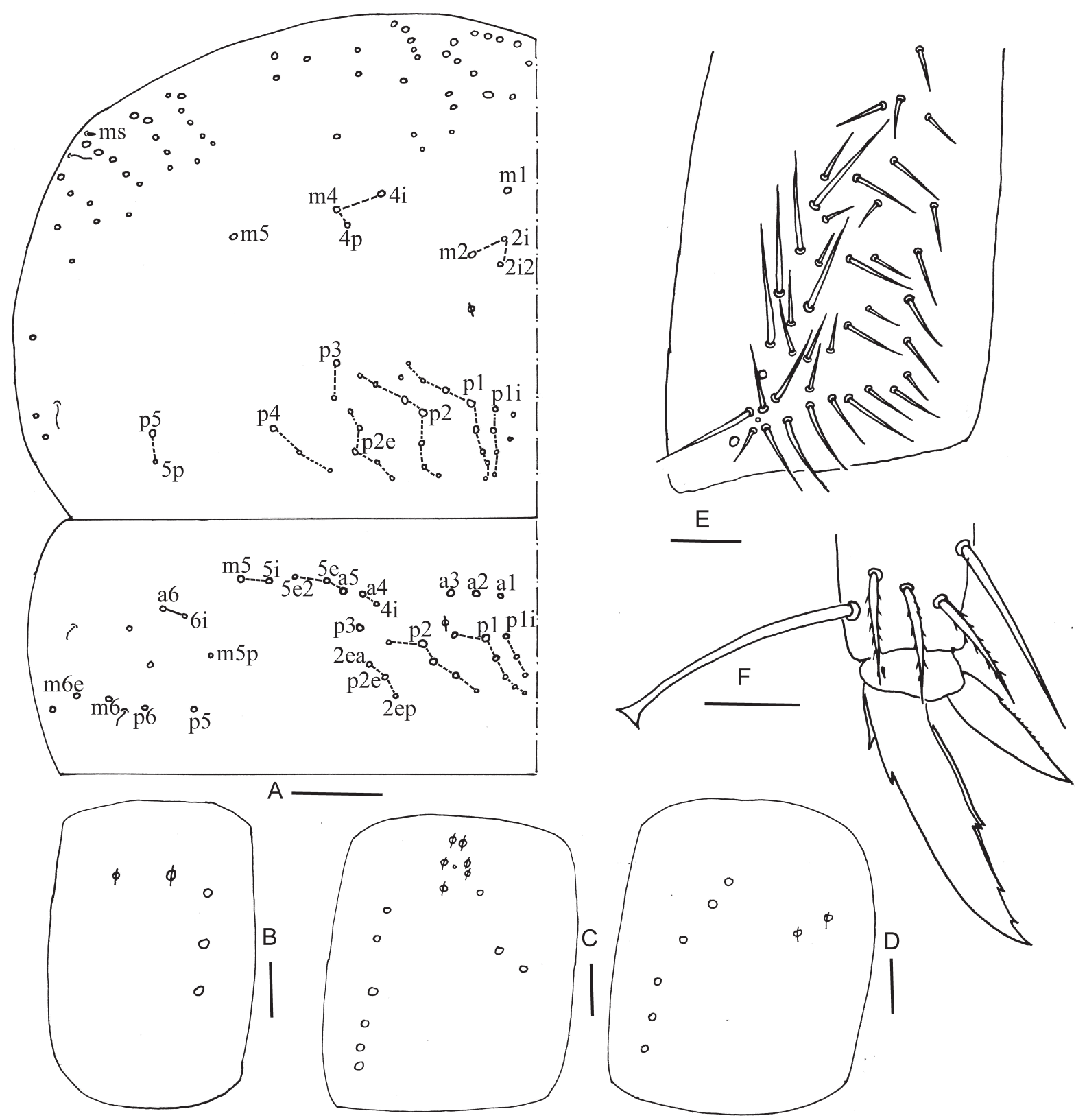

Fig. 3. Entomobrya leviseta sp. nov. A. Chaetotaxy of Th. II-III. B. Coxal macrochaetal formula of fore leg. C. Coxal macrochaetal formula of middle leg. D. Coxal macrochaetal formula of hind leg. E. Trochanteral organ. F. Hind foot complex. Scale bars: A $=50 \mu \mathrm{m} ; \mathrm{B}-\mathrm{F}=20 \mu \mathrm{m}$. 


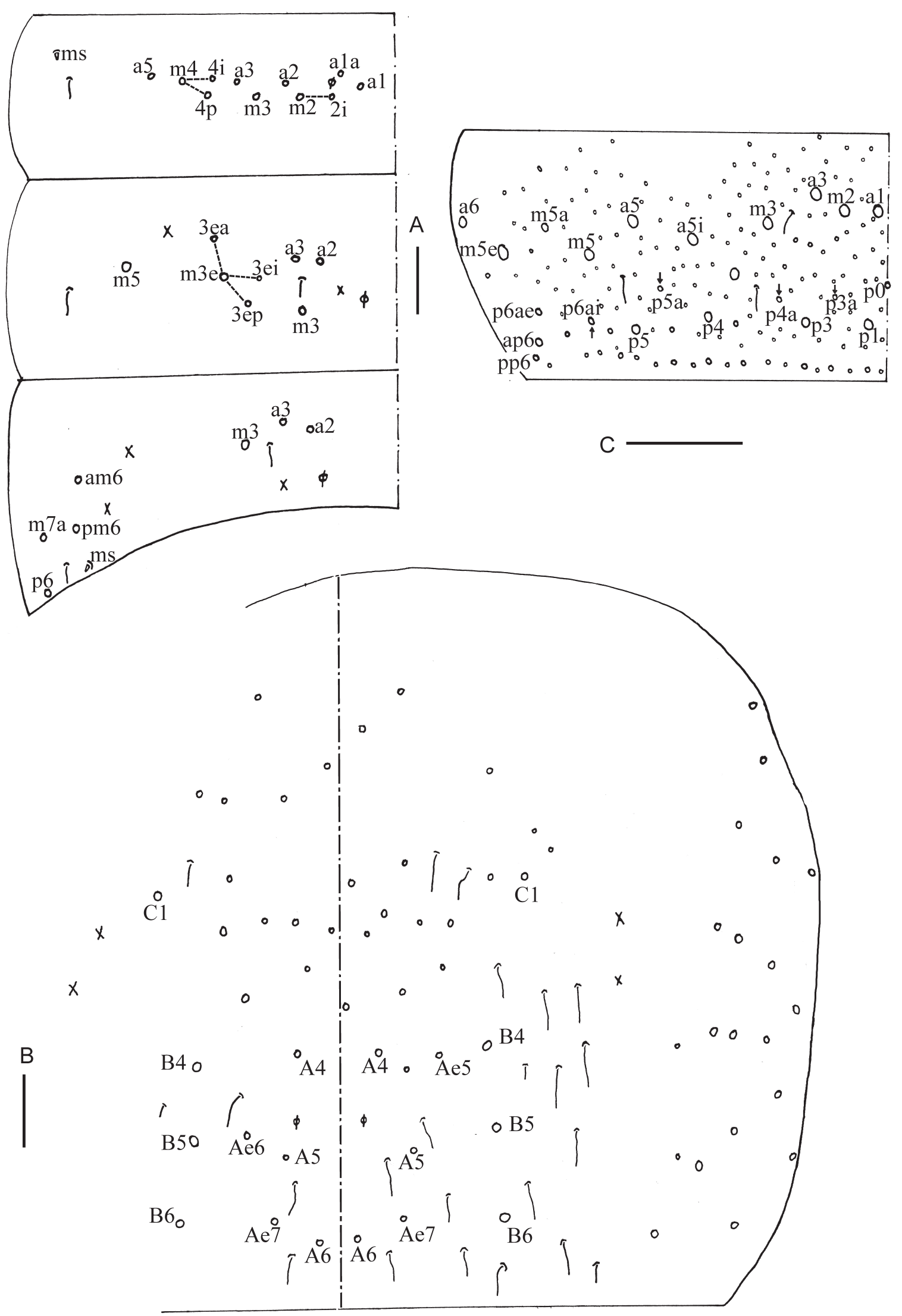

Fig. 4. Entomobrya leviseta sp. nov. A. Chaetotaxy of Abd. I-III. B. Chaetotaxy of Abd. IV. C. Chaetotaxy of Abd. V. Scale bars: A-C $=50 \mu \mathrm{m}$. 
Thorax. Th. II with 4 (m1, m2, m2i, m2i2) medio-medial, 3 (m4, m4i, m4p) medio-sublateral, 31-34 posterior mac, $1 \mathrm{~ms}$ and 2 sens. Th. III with 38-43 (46) mac and 2 sens (Fig. 3A). Coxal macrochaetal formula as 3 (2 pseudopores)/4+1-2, 3 (5-6 pseudopores)/4+2 (2 pseudopores) (Fig. 3B-D). Trochanteral organ with 43-44 (61) chaetae (Fig. 3E). Tenent hair subequal to inner side of unguis, with tip strongly clavate. Unguis with 4 inner teeth, basal pair located $0.44-0.49$ distance from base of inner edge of unguis, distal unpaired two respectively at $0.68-0.73$ and $0.85-0.87$ distance from base. Unguiculus acuminate with outer edge serrate (Fig. 3F).

Abdomen. Abd. IV 5.31-7.55 times as Abd. III in length along dorsal midline. Abd. I with 11(10) (m2i, $\mathrm{m} 2, \mathrm{~m} 3, \mathrm{~m} 4, \mathrm{~m} 4 \mathrm{i}, \mathrm{m} 4 \mathrm{p}, \mathrm{a} 1, \mathrm{a} 1 \mathrm{a}, \mathrm{a} 2, \mathrm{a} 3, \mathrm{a} 5, \mathrm{~m} 4 \mathrm{i}$ sometimes absent) mac, $1 \mathrm{~ms}$ and 1 sens; sens inner to ms. Abd. II with 6 (7) (a2, a3, m3e, m3ea, m3ep, m3, m3ei sometimes present) central, 1 (m5) lateral mac and two sens. Abd. III with 3 (a2, a3, m3) central, 4 (am6, pm6, m7a, p6) lateral mac, $1 \mathrm{~ms}$ and 2 sens (Fig. 4A). Abd. IV with 33-46 central and 20-23 lateral mac (Fig. 4B). Abd. V with 3 sens (Fig. 4C). Ventral tube with 15-23 ciliate chaetae on anterior face (Fig. 5A), 2 apical smooth and many ciliate chaetae on posterior face (Fig. 5B), 6 smooth and 8-13 ciliate chaetae on each lateral flap (Fig. 5C). Manubrial plaque with 8-14 ciliate chaetae and 3 pseudopores on each side (Fig. 5D). Distal

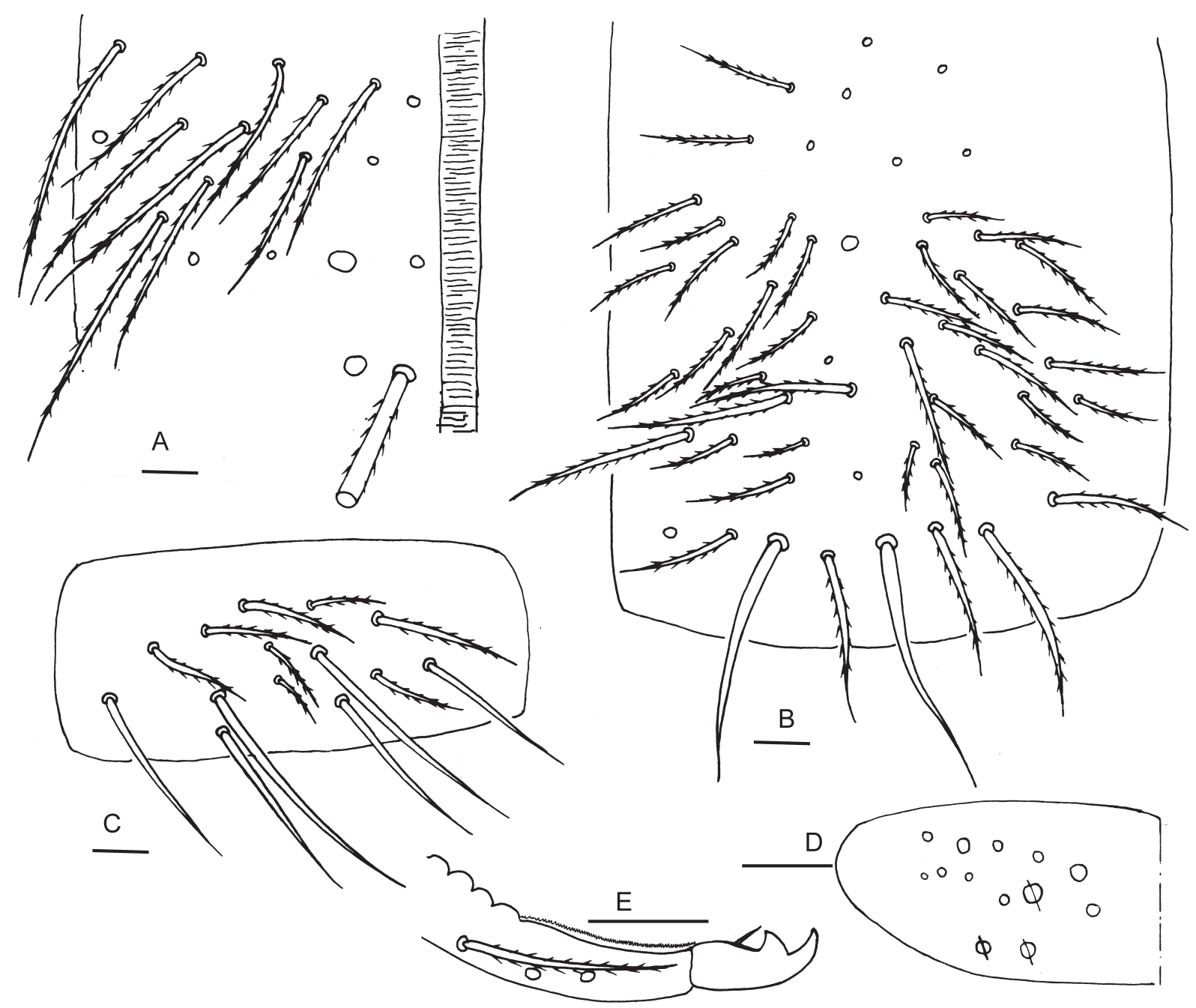

Fig. 5. Entomobrya leviseta sp. nov. A. Anterior face of ventral tube. B. Posterior face of ventral tube. C. Lateral flap of ventral tube. D. Plaque of manubrium. E. Distal part of dens and mucro. Scale bars: $\mathrm{A}-\mathrm{E}=20 \mu \mathrm{m}$. 
smooth part of dens about 1.00-1.28 times as long as mucro. Mucro bidentate and subapical tooth equal to or slightly larger than apical tooth. Basal spine almost reaching apex of subapical tooth (Fig. 5E).

\section{Remarks}

The prelabral chaetae on labrum are ciliated in Entomobrya generally (Jordana 2012) or finely ciliate (Katz et al. 2015), but they are smooth in E. striatella Börner, 1909 (Jordana 2012; Yosii et al. 1963). The new species is the second species of Entomobrya with smooth prelabral chaetae and it is also similar to E. striatella in colour pattern on Th. II, with three longitudinal stripes, but the colour pattern on Th. III, Abd. IV-V and chaetotaxy on Abd. I \& IV are different (Table 1).

\section{Entomobrya polychaeta sp. nov.}

urn:1sid:zoobank.org:act:B53D28DE-4033-467F-9077-60C916C12AF7

Figs 6-10, Table 2

\section{Diagnosis}

Three pairs of interrupted, longitudinal dark blue stripes present from Th. II to Abd. III; 7-9 mediomedial and 6-8 medio-sublateral mac on Th. II; 4 central and 8 lateral mac on Abd. III.

\section{Etymology}

The specific epithet is derived from the Latin 'poly', meaning 'many', referring to the many mac on the lateral side of Abd. III.

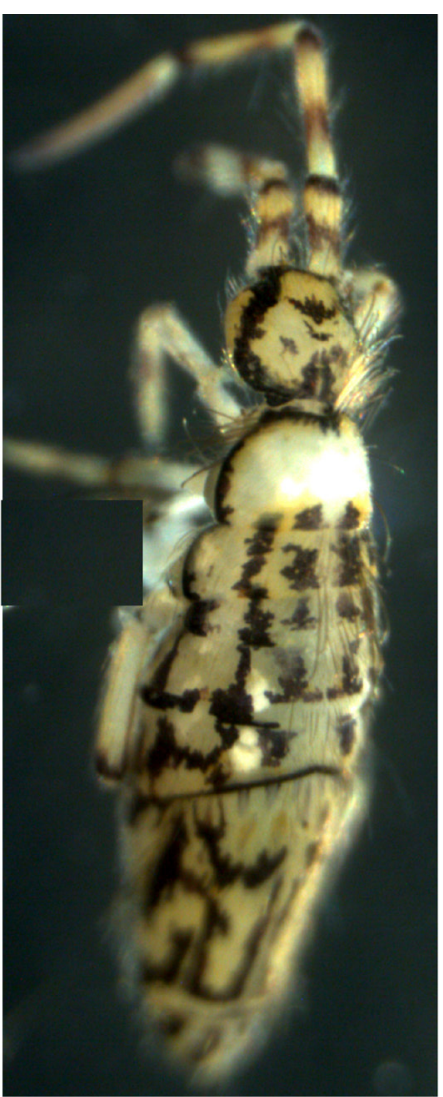

A

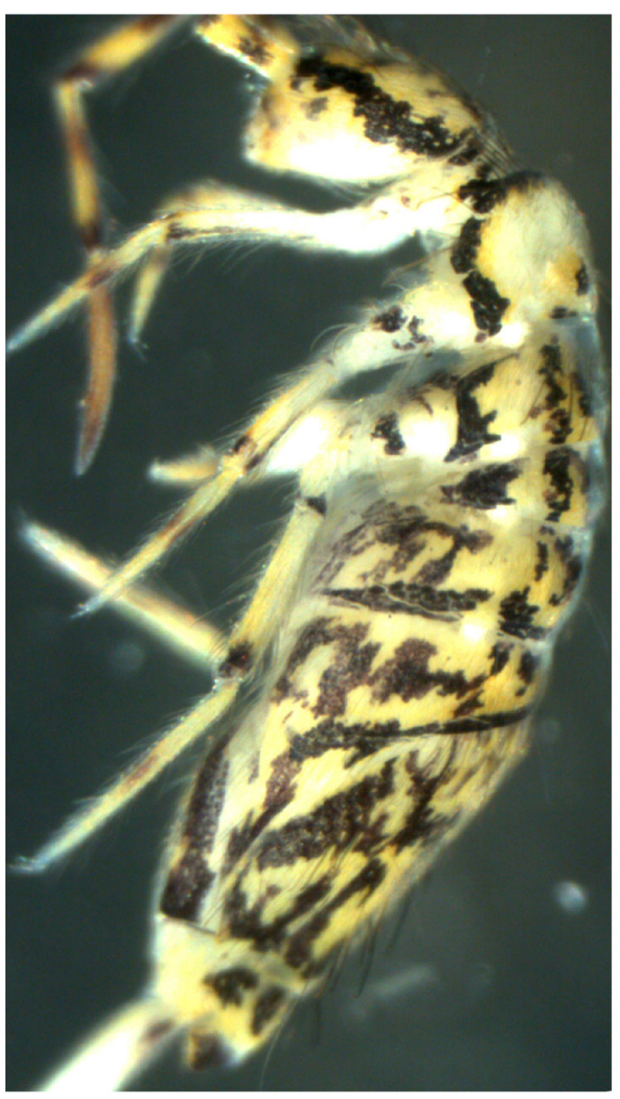

$\mathrm{B}$

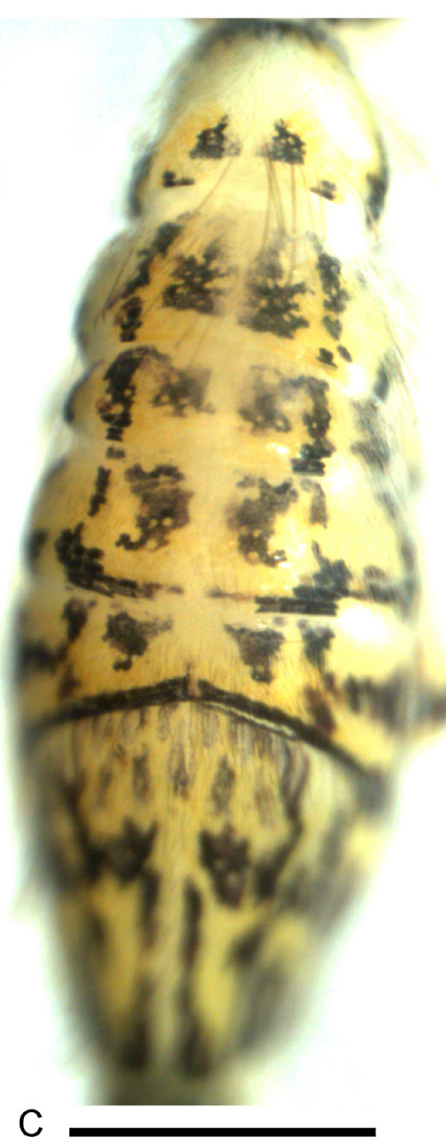

C

Fig. 6. Entomobrya polychaeta sp. nov. A-C. Habitus. Scales bars: A-C = 500 $\mu \mathrm{m}$. 
Table 2. Comparison among Entomobrya polychaeta sp. nov., E. koreana Yosii, 1965 and E. retingensis Baquero \& Jordana, 2008.

\begin{tabular}{cccc}
\hline Characters & $\begin{array}{c}\text { E. polychaeta } \\
\text { sp. nov. }\end{array}$ & E. koreana & E. retingensis \\
\hline Blue longitudinal stripes from & 6 stripes & 5 stripes & no longitudinal stripes \\
Th. II to Abd. III & $7-9$ & 2 & 0 \\
Medio-medial mac on Th. II & $6-8$ & 4 & 2 \\
Medio-lateral mac on Th. II & 4 & 3 & 3 \\
Central mac on Abd. III & 8 & 4 & 3 \\
Lateral mac on Abd. III & $1.5-1.6$ & 2.3 & 2.4 \\
Length ratio of smooth part of & & Korea & China (Tibet) \\
dens to mucro & China (Shaanxi) & &
\end{tabular}
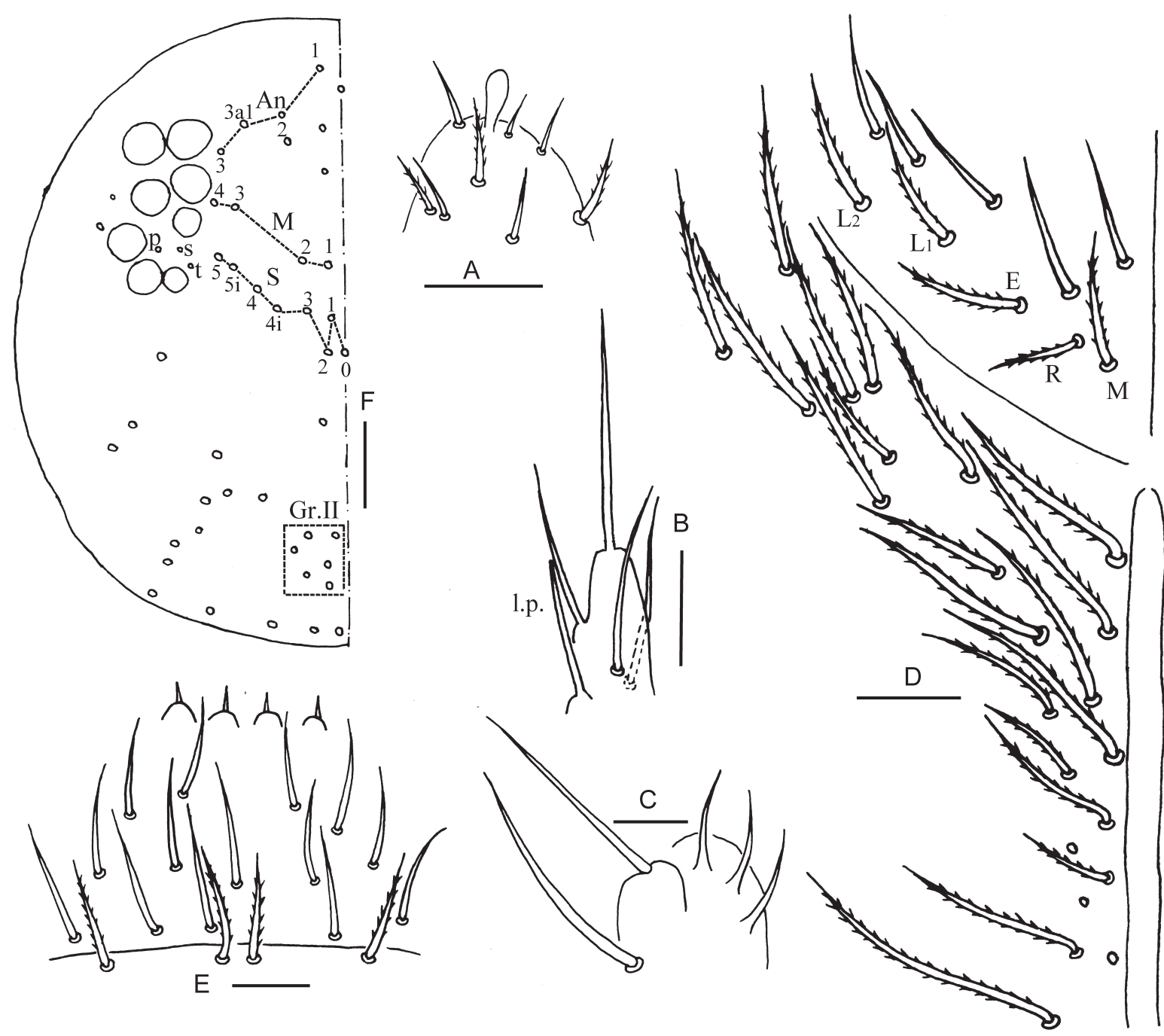

Fig. 7. Entomobrya polychaeta sp. nov. A. Apex of Ant. IV. B. Lateral process of labial palp. C. Maxillary outer lobe. D. Labium. E. Labrum. F. Dorsal chaetotaxy of head. Scale bars: A-C, E, $=20 \mu \mathrm{m} ; \mathrm{D}, \mathrm{F}=$ $50 \mu \mathrm{m}$. 


\section{Type material}

\section{Holotype}

CHINA: + on slide, 108 National Highway, Mazhao Town, Zhouzhi County, Xi'an City, Shaanxi Province, $34^{\circ} 03^{\prime} 35^{\prime \prime} \mathrm{N}, 108^{\circ} 11^{\prime} 47^{\prime \prime} \mathrm{E}$, altitude ca $566 \mathrm{~m}$, in litter, with aspirator, 15 Jul. 2012, Yitong Ma leg. (Nantong University, collection number 1115).

\section{Paratypes}

CHINA: 2 $q$ on slides, same collecting data as for holotype.

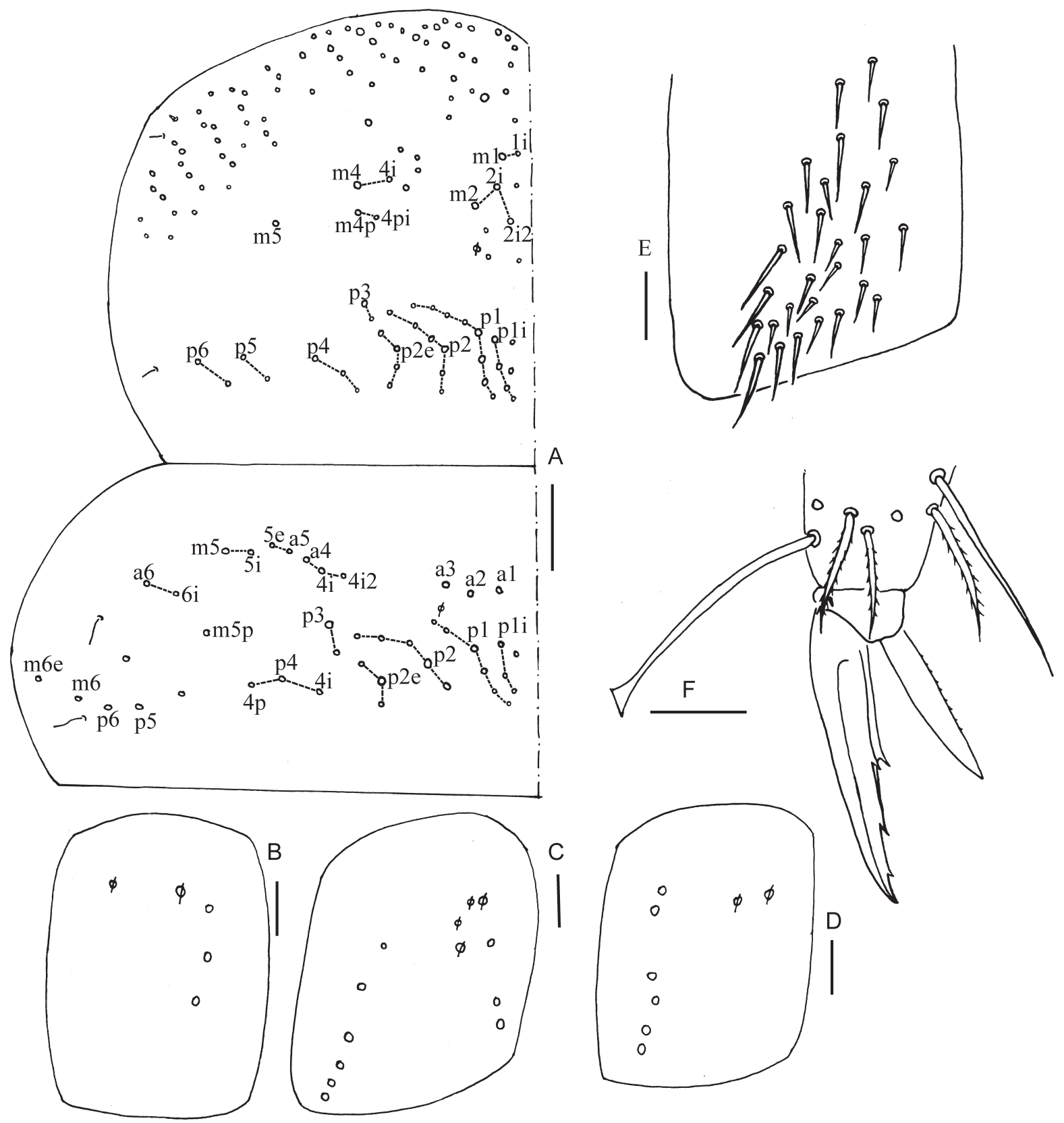

Fig. 8. Entomobrya polychaeta sp. nov. A. Chaetotaxy of Th. II-III. B. Coxal macrochaetal formula of fore leg. C. Coxal macrochaetal formula of middle leg. D. Coxal macrochaetal formula of hind leg. E. Trochanteral organ. F. Hind foot complex. Scale bars: A $=50 \mu \mathrm{m} ; \mathrm{B}-\mathrm{F}=20 \mu \mathrm{m}$. 


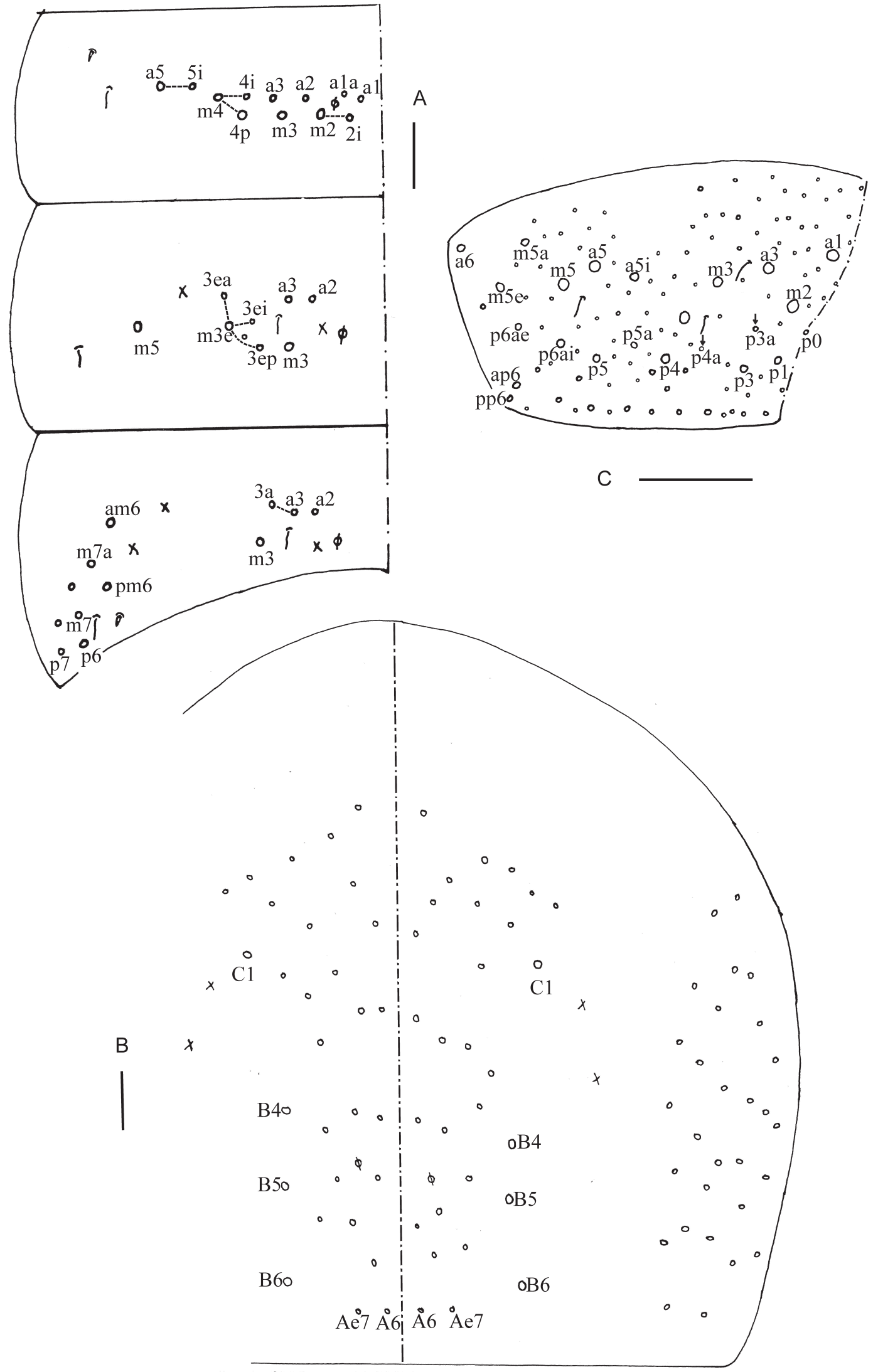

Fig. 9. Entomobrya polychaeta sp. nov. A. Chaetotaxy of Abd. I-III. B. Chaetotaxy of Abd. IV. C. Chaetotaxy of Abd. V. Scale bars: A-C $=50 \mu \mathrm{m}$. 


\section{Description}

MeAsurements. Body length up to $2.24 \mathrm{~mm}$.

CoLour. Ground colour pale yellow. Middle and distal parts of Ant. I-III blue pigmented and pigment also present on Ant. IV. An irregular dark blue circle present along margin of head. Besides a transverse dark stripe present between eyepatches, some dark patches present on middle and posterior part of head. A pair of longitudinal stripes present laterally from anterior part of Th. II to Abd. IV. There are also two pairs of discontinuous longitudinal stripes from posterior part of Th. II to Abd. III: one is located centrally and other medio-laterally. Posterior margin of Abd. III with a narrow transverse blue stripe. Abd. IV with longitudinal median stripes and irregular patches. Coxae, distal part of femora and middle part of tibiotarsi with blue pigment (Fig. 6A-C).

HeAD. Antenna $0.58-0.68$ times as long as body. Antennal segments ratio as I: II : III : IV $=1.00: 1.56-$ $1.85: 1.32-1.74: 1.85-2.12$. Distal part of Ant. IV with many sensory chaetae, normal ciliate chaetae and apical bulb unilobed (Fig. 7A). Lateral process (1.p.) of labial palp curved with tip reaching apex of same papilla (Fig. 7B). Subapical chaeta of maxillary outer lobe subequal to apical one, 3 smooth hairs on sublobal plate (Fig. 7C). Labial chaetae $\mathrm{MREL}_{1} \mathrm{~L}_{2}$, all ciliate; chaeta $\mathrm{R} 0.67-0.70$ as long as chaeta $\mathrm{M}$ (Fig. 7D). Labral chaetae 4/5, 5, 4, prelabral chaetae ciliate and other smooth; labral papillae 4 and each with 1 minute denticle (Fig. 7E). Clypeus not clearly seen. Dorsal cephalic chaetotaxy with 4 antennal (An), 4 median (M), 8 sutural (S) mac and 6-7 mac in Gr. II; interocular chaetae as p, s, t (Fig. 7F).
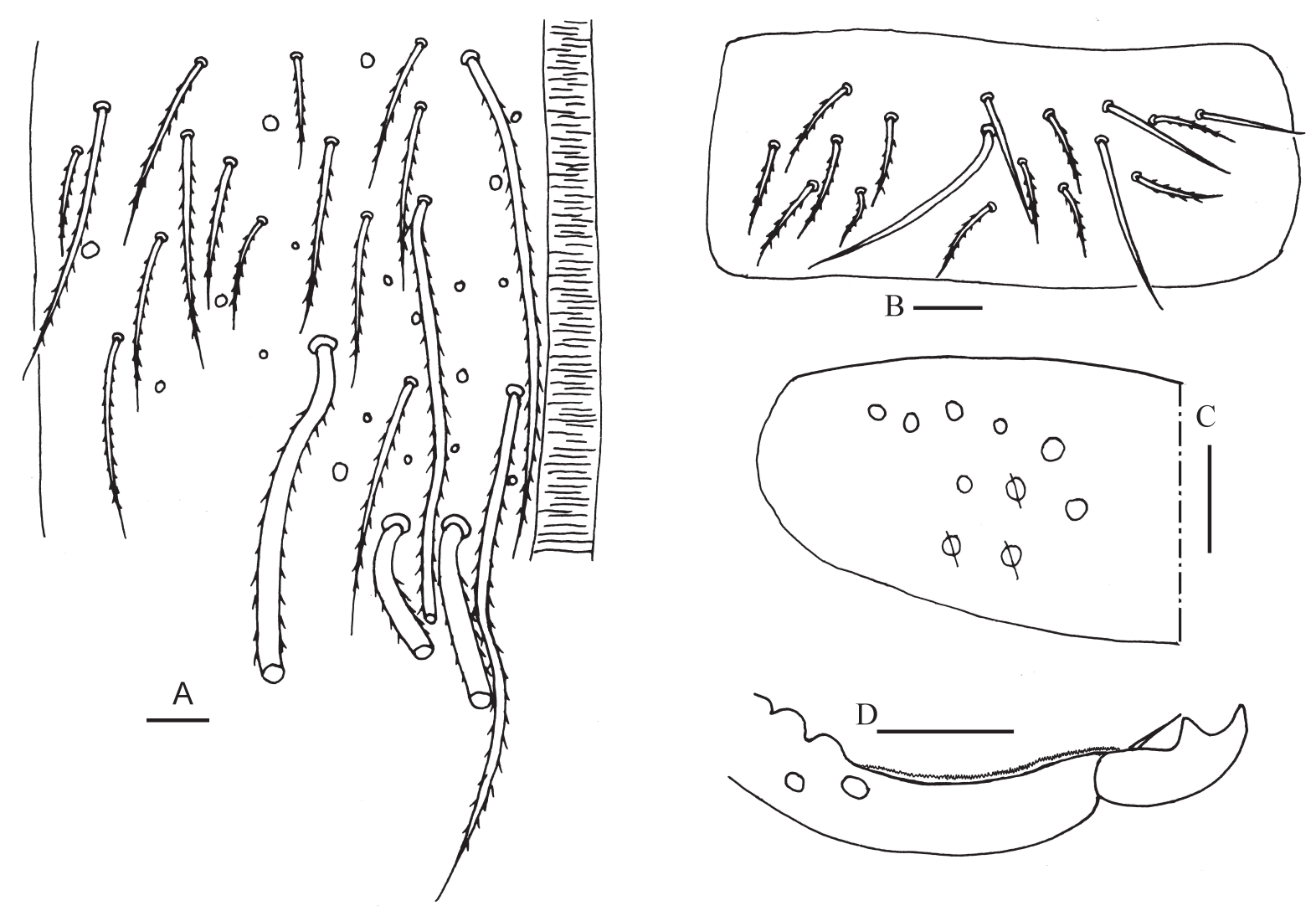

Fig. 10. Entomobrya polychaeta sp. nov. A. Anterior face of ventral tube. B. Lateral flap of ventral tube. C. Plaque of manubrium. D. Distal part of dens and mucro. Scale bars: A-D $=20 \mu \mathrm{m}$. 
Thorax. Th. II with 7-9 medio-medial, 6-8 medio-sublateral, 33-35 posterior mac, $1 \mathrm{~ms}$ and 2 sens. Th. III with 42-43 mac and 2 sens (Fig. 8A). Coxal macrochaetal formula as 3 ( 2 pseudopores) $/ 4+2,3$ (4 pseudopores) $/ 4+2$ ( 2 pseudopores) (Fig. 8B-D). Trochanteral organ with $28-33$ chaetae (Fig. 8E). Tenent hair subequal to inner side of unguis, with tip strongly clavate. Unguis with 4 inner teeth, basal pair located 0.47-0.49 distance from base of inner edge of unguis, distal unpaired two respectively at $0.71-0.73$ and $0.88-0.89$ distance from base. Unguiculus acuminate with outer edge serrate (Fig. 8F).

Abdomen. Abd. IV 5.02-6.11 times as Abd. III in length along dorsal midline. Abd. I with 12(11) (m2i, $\mathrm{m} 2, \mathrm{~m} 3, \mathrm{~m} 4, \mathrm{~m} 4 \mathrm{i}, \mathrm{m} 4 \mathrm{p}, \mathrm{a} 1, \mathrm{a} 1 \mathrm{a}, \mathrm{a} 2, \mathrm{a} 3, \mathrm{a} 5, \mathrm{~m} 4 \mathrm{i}$ sometimes absent) mac, $1 \mathrm{~ms}$ and 1 sens; sens inner to ms. Abd. II with 7(8) (a2, a3, m3e, m3ea, m3ei, m3ep, m3, an unknown mac sometimes present between m3e and m3ep) central, 1 (m5) lateral mac and two sens. Abd. III with 4 (a2, a3, a3a, m3) central, 8(6 or 7) (am6, pm6, m7a, m7, p6, p7, 2 unknown) lateral mac, 1 ms and 2 sens (Fig. 9A). Abd. IV with 48-58 central and 26-29 lateral mac (Fig. 9B). Abd. V with 3 sens (Fig. 9C). Ventral tube with 39 ciliate chaetae on anterior face (in 1 individual) (Fig. 10A), posterior face not clearly seen, 5 smooth and 11-17 ciliate chaetae on each lateral flap (Fig. 10B). Manubrial plaque with 7 ciliate chaetae and 3 pseudopores on each side in 2 individuals (Fig. 10C). Distal smooth part of dens about 1.48-1.56 times as long as mucro. Mucro bidentate and subapical tooth equal to apical tooth. Basal spine almost reaching apex of subapical tooth (Fig. 10D).

\section{Remarks}

The new species is characterised by its three pairs of longitudinal dark blue stripes present from Th. II to Abd. III, 7-9 medio-medial and 6-8 medio-lateral mac on Th. II, usually 8 lateral mac on Abd. III. It is most similar to the Korean species E. koreana Yosii, 1965 and the Chinese E. retingensis Baquero \& Jordana, 2008 in colour pattern, but the differences between them are great, such as chaetotaxy on Th. II and Abd. III and the ratio of the smooth part of the dens to the mucro (Table 2).

$$
\begin{gathered}
\text { Entomobrya dingi sp. nov. } \\
\text { urn:1sid:zoobank.org:act:8B3E500B-6261-4944-9A11-D22FE05D1B82 }
\end{gathered}
$$

Figs 11-15, Table 3

\section{Diagnosis}

Ground colour yellowish and no obvious patches or stripes; head with 8 mac in sutural area and 8-9 mac in Gr. II; 8-10 mac on Abd. I; 5 central mac on Abd. II \& III, 18-26 central mac on Abd. IV.

\section{Etymology}

The new species is named after Fudong Ding, who collected the specimens.

\section{Type material}

\section{Holotype}

CHINA: $q$ on slide, Yuanlong Village, Yongfeng Town, Zhaoyang District, Zhaotong City, Yunnan Province, $27^{\circ} 17^{\prime} 25^{\prime \prime} \mathrm{N}, 103^{\circ} 39^{\prime} 09^{\prime \prime}$ E, in litter of maize, with aspirator, 1 Aug. 2014, Fudong Ding leg. (Nantong University, collection number 1149).

\section{Paratypes}

CHINA: $9+q$ on slides, same collecting data as for holotype.

\section{Description}

Measurement. Body length up to $1.66 \mathrm{~mm}$. 
Table 3. Comparison among Entomobrya dingi sp. nov., E. dungeri Jordana, Schulz \& Baquero, 2011, E. huangi Chen \& Ma, 1998 and E. lhotseae Yosii, 1971.

\begin{tabular}{lcccc}
\hline \multicolumn{1}{c}{ Characters } & E. dingi sp. nov. & E. dungeri & E. huangi & E. lhotseae \\
\hline S mac on head & present & absent & absent & absent \\
Denticle on labral papillae & present & absent & present & present \\
Mac on Abd. I & $8-10$ & unknown & 4 & 5 \\
Central mac on Abd. II & 5 & 5 & 4 & 4 \\
Central mac on Abd. III & 5 & 4 & 3 & 2 \\
Lateral mac on Abd. III & 3 & 6 & 2 & 3 \\
Central mac on Abd. IV & $18-26$ & 22 & 12 & 14 \\
Chaetae on manubrial plaque & $4-5$ & 4 & unknown & 2 \\
Locality & China (Yunnan) & Germany & China (Tibet) & Nepal \\
\hline
\end{tabular}

CoLour. Ground colour yellowish. Eyepatches dark blue. Blue pigment present on distal part of each segment of antenna and lateral side of Th. II-III (Fig. 11 A-B).

HEAD. Antenna 0.43-0.53 times as long as body. Antennal segments ratio as I: II : III: IV $=1.00: 1.67-$ $1.96: 1.60-1.83: 2.05-2.74$. Distal part of Ant. IV with many sensory chaetae, normal ciliate chaetae and apical bulb bilobed (Fig. 12A). Lateral process (1.p.) of labial palp curved with tip not reaching apex of same papilla (Fig. 12B). Subapical chaeta of maxillary outer lobe subequal to apical one, 3 smooth hairs on sublobal plate (Fig. 12C). Labial chaetae MREL $\mathrm{L}_{2}$, all ciliate; chaeta R $0.56-0.68$ as long as chaeta M (Fig. 12D). Labral chaetae 4/5, 5, 4, prelabral chaetae ciliate and other smooth; labral papillae 4 and each with 3-4 minute denticles (Fig. 12E). Clypeus with 14-16 ciliate chaetae (Fig. 12F). Dorsal cephalic chaetotaxy with 4 antennal (An), 4 median (M) and 8 sutural (S) mac, 8-9 mac in Gr. II (Fig. 12G); interocular chaetae as p, q, r, s, t (Fig. 12H).
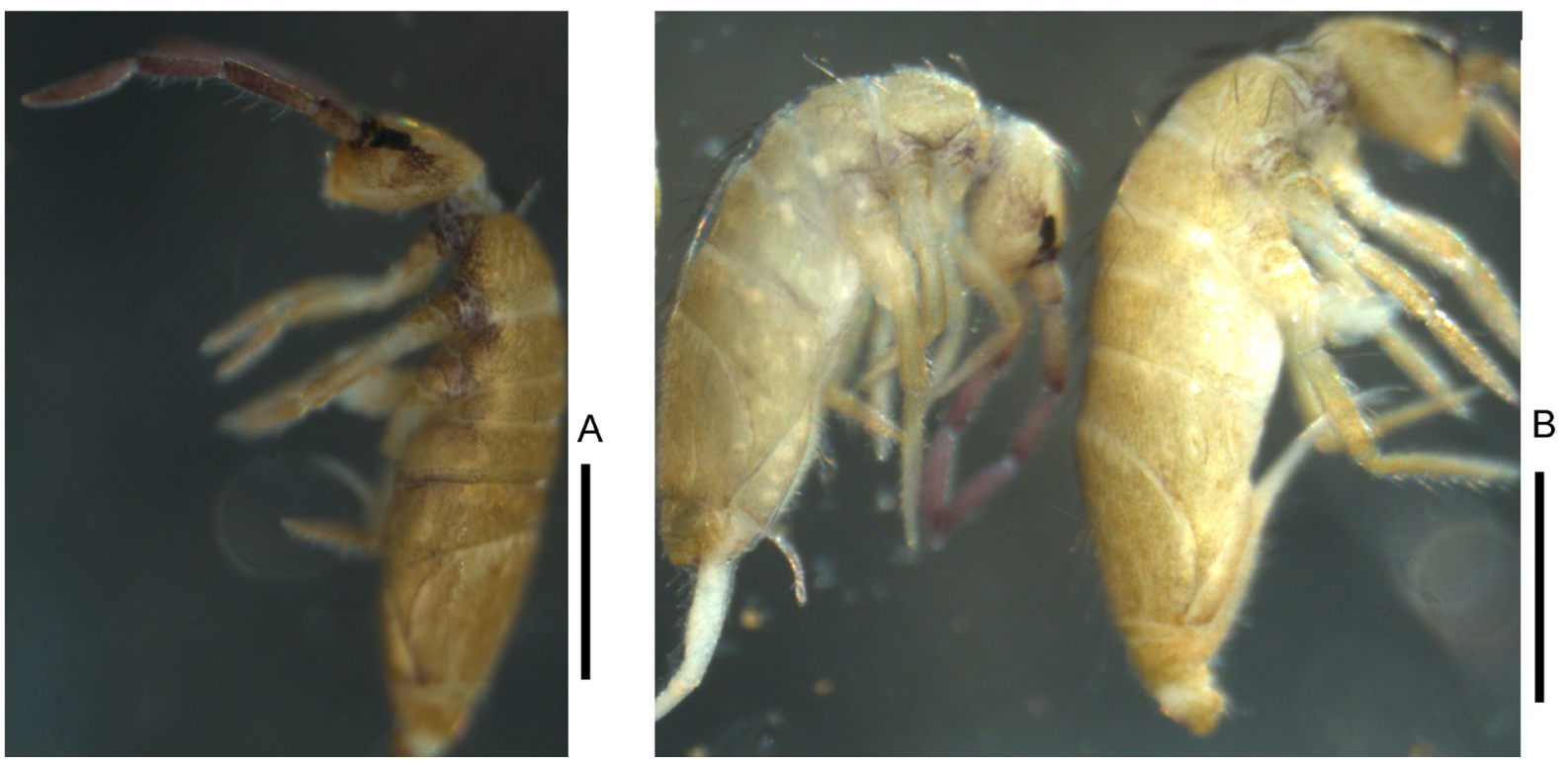

Fig. 11. Entomobrya dingi sp. nov. A-B. Habitus. Scales bar: A-B $=500 \mu \mathrm{m}$. 
Thorax. Th. II with 2(3) (m1, m2, m2i rarely present) medio-medial, 2 (m4, m4i) medio-sublateral, 19-27 posterior mac, $1 \mathrm{~ms}$ and 2 sens. Th. III with 27-31(34) mac and 2 sens (Fig. 13A). Coxal macrochaetal formula as 2 ( 2 pseudopores) / 4+1, 2-3 (2 pseudopores) / 4+2 (2 pseudopores) (Fig. 13BD). Trochanteral organ with 13-22 chaetae (Fig. 13E). Tenent hair slightly longer than inner side of unguis, with tip strongly clavate. Unguis with 4 inner teeth, basal pair located $0.45-0.50$ distance from base of inner edge of unguis, distal unpaired two respectively at $0.68-0.73$ and $0.84-0.87$ distance from base. Unguiculus acuminate with outer edge serrate (Fig. 13F).

ABdomen. Abd. IV 2.82-3.85 times as Abd. III in length along dorsal midline. Abd. I with 8-10 (m2, $\mathrm{m} 3, \mathrm{~m} 4, \mathrm{~m} 4 \mathrm{i}, \mathrm{m} 4 \mathrm{p}, \mathrm{a} 1, \mathrm{a} 2, \mathrm{a} 3, \mathrm{a} 5, \mathrm{a} 5 \mathrm{i}, \mathrm{m} 4 \mathrm{i}$ and a5i sometimes absent) mac, $1 \mathrm{~ms}$ and 1 sens; sens inner
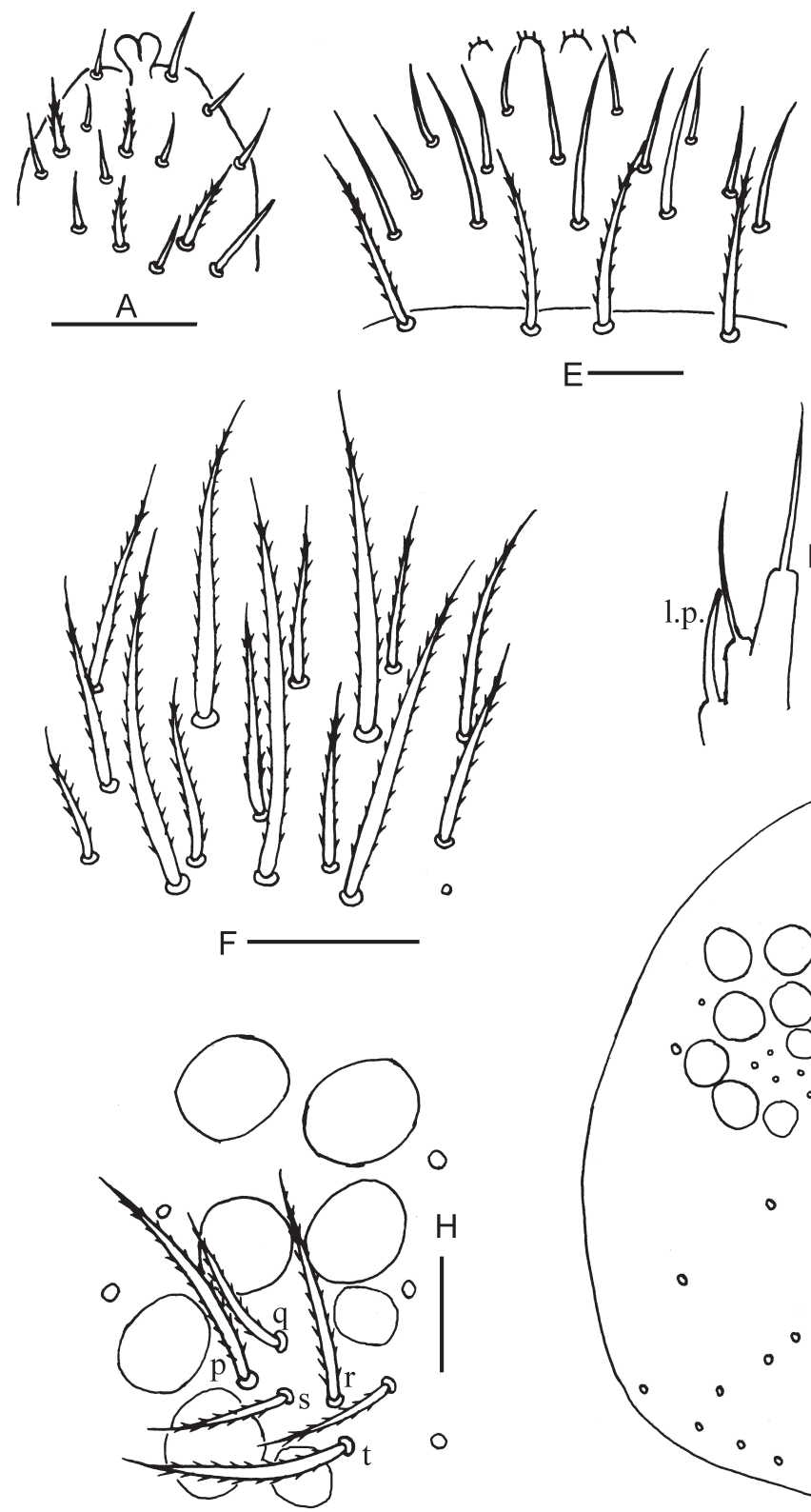
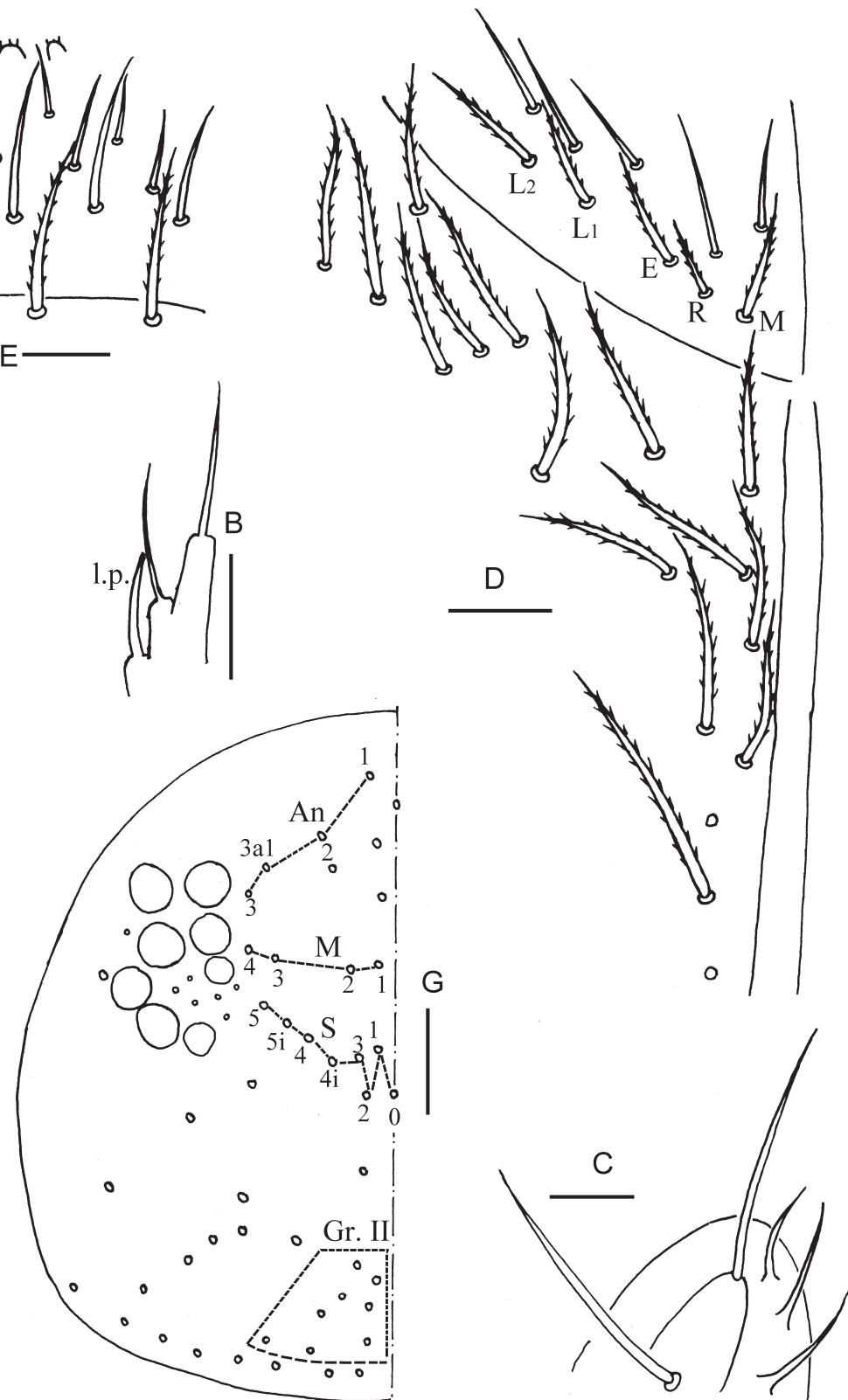

Fig. 12. Entomobrya dingi sp. nov. A. Apex of Ant. IV. B. Lateral process of labial palp. C. Maxillary outer lobe. D. Labium. E. Labrum. F. Clypeal chaetae. G. Dorsal chaetotaxy of head. H. Eyes and interocular chaetotaxy. Scale bars: A-C, E-F, H=20 $\mu \mathrm{m} ; \mathrm{D}, \mathrm{G}=50 \mu \mathrm{m}$. 
to ms. Abd. II with 5 (a2, a3, m3e, m3ep, m3) central, 1 (m5) lateral mac and and 2 sens. Abd. III with 5 (a1, a2, a3, m3, m3e) central, 3 (am6, pm6, p6) lateral mac and $1 \mathrm{~ms}$ and 2 sens (Fig. 14A). Abd. IV with 18-26 central and 7-12 lateral mac (Fig. 14B). Abd. V with 3 sens (Fig. 14C-D). Ventral tube with 14-16 ciliate chaetae on anterior face (Fig. 15A), 2 smooth and 9-14(6) ciliate or much ciliate chaetae on posterior face (Fig. 15B), 3-4 smooth and 4-7 ciliate chaetae on each lateral flap (Fig. 15C). Manubrial plaque with 4-5 ciliate chaetae and 2 pseudopores on each side (Fig. 15D). Distal smooth part of dens about 2.67-3.70 times as long as mucro. Mucro bidentate and subapical tooth smaller than or equal to apical tooth. Basal spine almost reaching apex of subapical tooth (Fig. 15E).

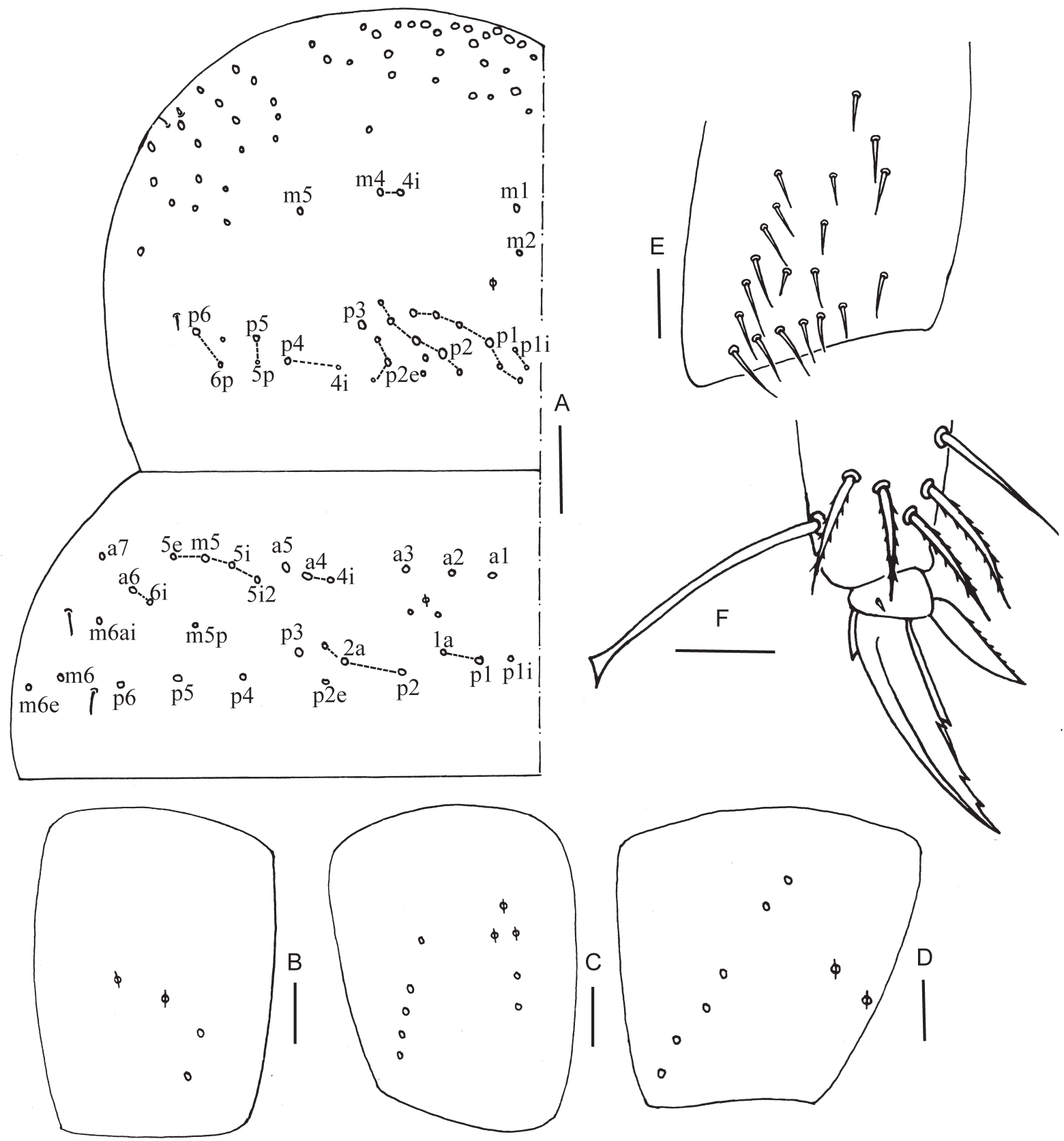

Fig. 13. Entomobrya dingi sp. nov. A. Chaetotaxy of Th. II-III. B. Coxal macrochaetal formula of fore leg. C. Coxal macrochaetal formula of middle leg. D. Coxal macrochaetal formula of hind leg. E. Trochanteral organ. F. Hind foot complex. Scale bars: A $=50 \mu \mathrm{m} ; \mathrm{B}-\mathrm{F}=20 \mu \mathrm{m}$. 
MA Y. \& SHI S., Three new species of Entomobrya from China

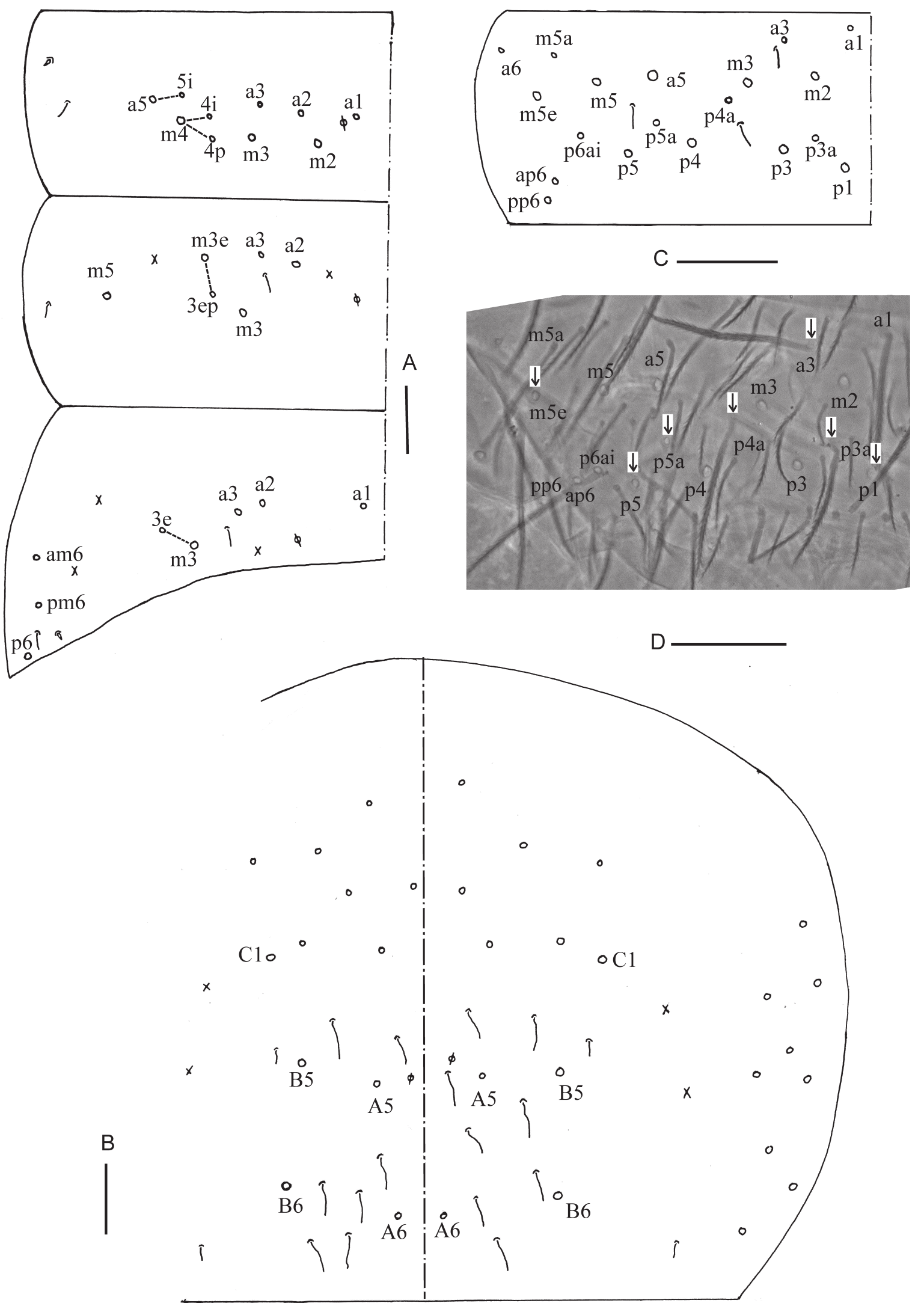

Fig. 14. Entomobrya dingi sp. nov. A. Chaetotaxy of Abd. I-III. B. Chaetotaxy of Abd. IV. C-D. Chaetotaxy of Abd. V (arrows pointing to mac). Scale bars: A-D $=50 \mu \mathrm{m}$. 


\section{Remarks}

The colour pattern of the new species is simple and there is only a little pigment on the body. It is similar to E. dungeri Jordana, Schulz \& Baquero, 2011, E. lhotseae Yosii, 1971 and E. huangi Chen \& Ma, 1998 in colour pattern, but can be distinguished by the following characters: presence of $\mathrm{S}_{1}$ on head, $8-10 \mathrm{mac}$ on Abd. I, 5 central mac on Abd. II \& III respectively and 18-26 central mac on Abd. IV (Table 3).

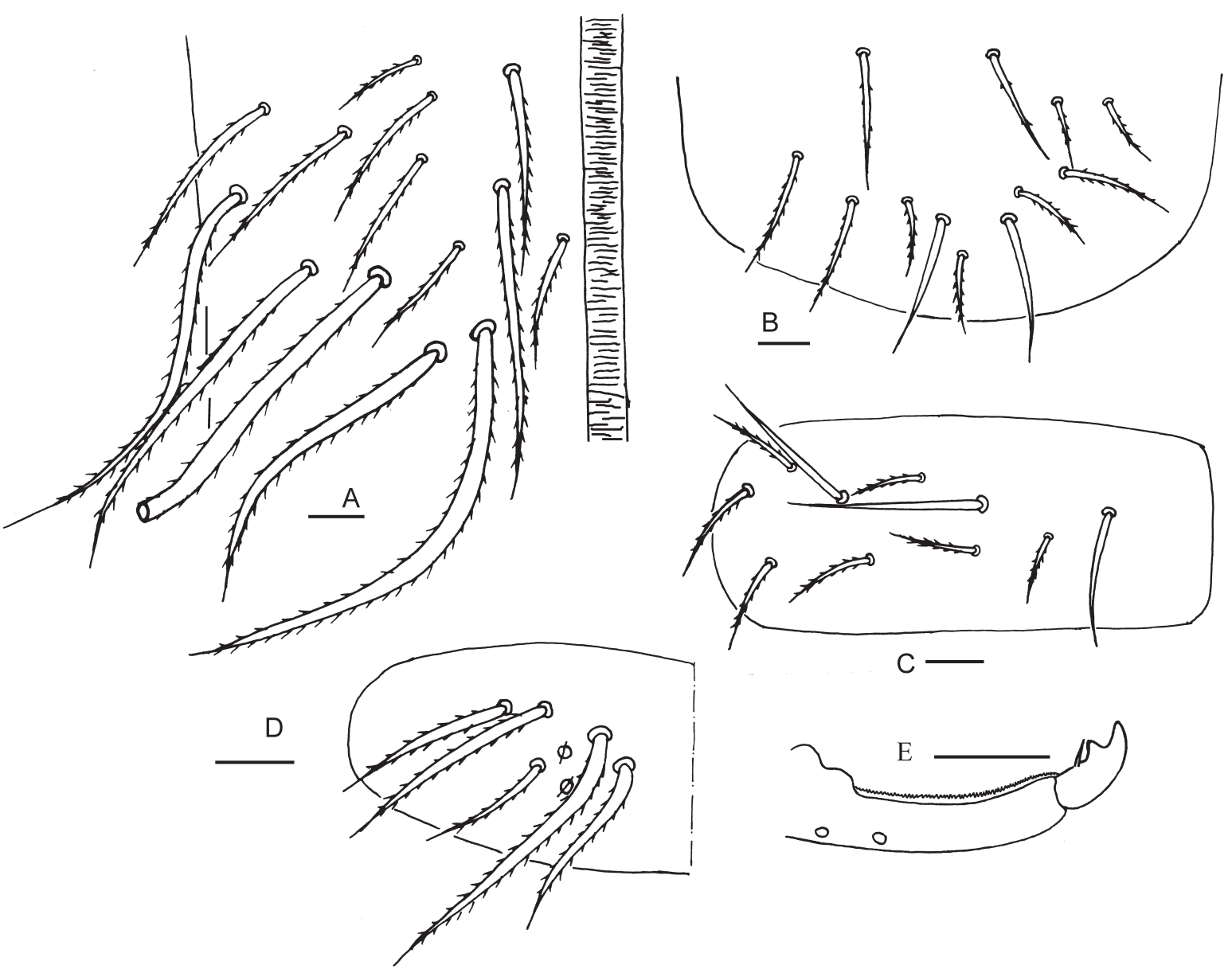

Fig. 15. Entomobrya dingi sp. nov. A. Anterior face of ventral tube. B. Posterior face of ventral tube. C. Lateral flap of ventral tube. D. Plaque of manubrium. E. Distal part of dens and mucro. Scale bars: A-E $=20 \mu \mathrm{m}$.

\section{Key to the Chinese species of the genus Entomobrya}

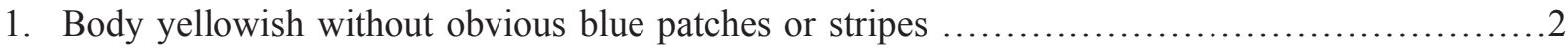

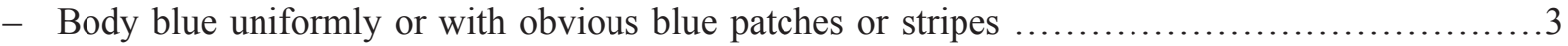

2. Abd. I with 8-10 mac E. dingi sp. nov.

- Abd. I with 4 mac E. huangi Chen \& Ma, 1998

3. Body blue uniformly except head E. griseoolivata (Packard, 1873)

- Body with obvious blue patches or stripes 


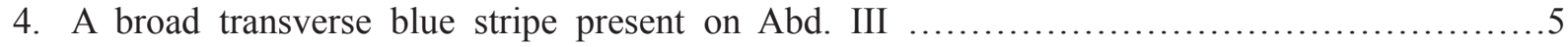

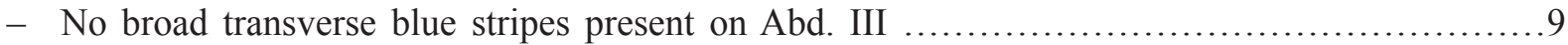

5. No transverse blue stripes present on Th. III ................E. aino (Matsumura \& Uchida, 1931)

- A transverse blue stripe present on Th. III ...............................................6

6. Posterior half of Abd. IV dark blue .............................E. dorsosignata Stach, 1963

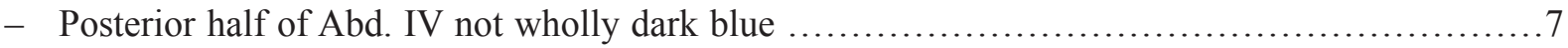

7. A pair of small patches present on anterior of Abd. IV .............E. pekinensis Stach, 1963

- No pair of patches present on anterior of Abd. IV ........................................... 8

8. A narrow blue stripe present on posterior margin of Th. II ........E. corticalis (Nicolet, 1842)

- No stripes present on posterior margin of Th. II ......................E. imitabilis Stach, 1963

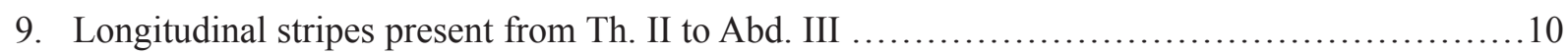

- No longitudinal stripes present from Th. II to Abd. III ...................................... 12

10. Th. II with $7-9$ medio-medial mac ..................................... polychaeta sp. nov.

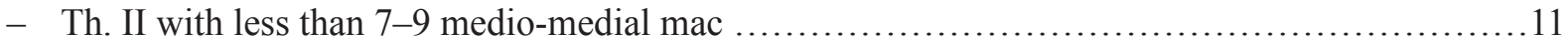

11. Four mac present on Abd. III centrally ...............E. fjellbergi Baquero \& Jordana, 2008

- Three mac present on Abd. III centrally ..............E. karlstetterae Baquero \& Jordana, 2008

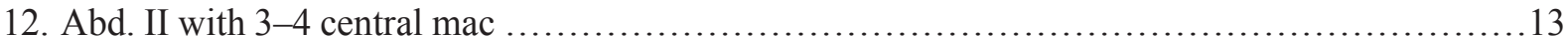

- Abd. II with 6-9 central mac .............................................................. 15

13. Th. II with 2 medio-medial mac ............................... marginata (Tullberg, 1871)

- Th. II without medio-medial mac ......................................................... 14

14. Abd. III with 2 central mac .........................E. chungseensis Baquero \& Jordana, 2008

- Abd. III with 4 central mac ..........................E. mieheorum Baquero \& Jordana, 2008

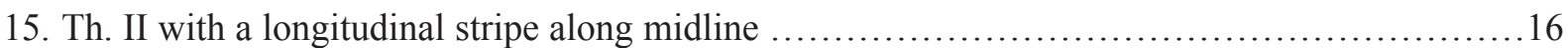

- Th. II with no longitudinal stripe along midline ............................................. 17

16. Irregular patches present on Abd. I-III respectively ...E. retingensis Baquero \& Jordana, 2008

- No irregular patches present on Abd. I-III .............................. leviseta sp. nov

17. A pair of oblique stripes present on Abd. II laterally ...............E. hortensis Stach, 1963

- No oblique stripes present on Abd. II laterally ...........E. cheni Baquero, Arbea \& Jordana, 2010

\section{Discussion}

The colour pattern is variable in Entomobrya and in most species completely pale specimens and very dark ones are found (Christiansen 1958). However, the latest studies indicate that specimens sharing similar colour patterns may be different species (Katz et al. 2015; Pan et al. 2015). From 270 known species of the genus, most were described mainly based on the colour pattern and some of them are undoubtedly erroneously determined. The colour pattern, combined with other useful characters, especially the dorsal chaetotaxy, is very important in classification.

The dorsal chaetotaxy, a key character in species delimitation, is weakly variable. The chaetotaxy on Abd. I and lateral part of Abd. III is interspecifically variable, but it is sometimes neglected. There are usually many chaetae on Abd. IV and it is very difficult to assess their homology. 


\section{Acknowledgements}

The study was supported by the Zhejiang Provincial Natural Science Foundation of China (LY15C040001) and the National Natural Science Foundation of China (36101880). Thanks are due to Feng Zhang (Nanjing Agricultural University), Zhixiang Pan (Taizhou University) and anonymous referees who provided kind advice on this manuscript.

\section{References}

Bellinger P.F., Christiansen K.A. \& Janssens F. 1996-2017. Checklist of the Collembola of the world. Available from http://www.collembola.org [accessed 20 Mar. 2017].

Chen J.X. \& Christiansen K.A. 1993. The genus Sinella with special reference to Sinella s. s. (Collembola: Entomobryidae) of China. Oriental Insects 27: 1-54. https://doi.org/10.1080/00305316.1993.10432236

Christiansen K.A. 1958. The Nearctic members of the genus Entomobrya (Collembola). Bulletin of the Museum of Comparative Zoology 118 (7): 439-545.

Gisin H. 1967. Espèces nouvelles et lignées évolutives de Pseudosinella endogés. Memórias e Estudos do Museu Zoológico da Universidade de Coimbra 301: 5-25.

Jordana R. 2012. Capbryinae and Entomobryini. In: Dunger W. \& Burkhardt U. (eds) Synopses on Palearctic Collembola. Soil Organisms 84 (1): 1-391.

Jordana R. \& Baquero E. 2005. A proposal of characters for taxonomic identification of Entomobrya species (Collembola, Entomobryomorpha), with description of a new species. Abhandlungen und Berichte des Naturkundemuseums Goerlitz 76 (2): 117-134.

Katz A.D., Giordano R. \& Soto-adames F.N. 2015. Operational criteria for cryptic species delimitation when evidence is limited, as exemplified by North American Entomobrya (Collembola: Entomobryidae). Zoological Journal of the Linnean Society 173 (4): 1-23. https://doi.org/10.1111/zoj.12220

Mari-Mutt J.A. 1986. Puerto Rican species of Lepidocyrtus and Pseudosinella (Collembola: Entomobryidae). Caribbean Journal of Science 22 (1-2): 1-48.

Pan Z.X., Zhang F. \& Li Y.B. 2015. Two closely related Homidia species (Entomobryidae, Collembola) revealed by morphological and molecular evidence. Zootaxa 3918 (2): 285-294.

https://doi.org/10.11646/zootaxa.3918.2.9

Stach J. 1963. The Apterygotan Fauna of Poland in Relation to the World-Fauna of this Group of Insects. Tribe: Entomobryini. Panstwowe Wydawnictwo Naukowe, Kraków.

Szeptycki A. 1979. Morpho-Systematic Studies on Collembola. IV. Chaetotaxy of the Entomobryidae and its Phylogenetical Significance. Polska Akademia Nauk, Zakład Zoologii Systematycznej I Doświadczalnej, Państwowe Wydawnictwo Naukowe, Warszawa, Kraków.

Yosii R. 1963. On some Collembola of Japan and adjacent countries. Contributions from the Biological Laboratory Kyoto University 19: 1-71.

Yosii R. \& Lee C.E. 1963. On some Collembola of Korea, with notes on the genus Ptenothrix. Contributions from the Biological Laboratory Kyoto University 15: 1-37.

Zhang F. \& Louis D. 2014. Systematic revision of Entomobryidae (Collembola) by integrating molecular and new morphological evidence. Zoologica Scripta 44: 298-311. 
Published on: 27 March 2018

Topic editor: Gavin Broad

Desk editor: Kristiaan Hoedemakers

Printed versions of all papers are also deposited in the libraries of the institutes that are members of the EJT consortium: Muséum national d'Histoire naturelle, Paris, France; Botanic Garden Meise, Belgium; Royal Museum for Central Africa, Tervuren, Belgium; Natural History Museum, London, United Kingdom; Royal Belgian Institute of Natural Sciences, Brussels, Belgium; Natural History Museum of Denmark, Copenhagen, Denmark; Naturalis Biodiversity Center, Leiden, the Netherlands; Museo Nacional de Ciencias Naturales-CSIC, Madrid, Spain; Real Jardín Botánico de Madrid CSIC, Spain. 